\title{
Potency preservation following stereotactic body radiation therapy for prostate cancer
}

Olusola Obayomi-Davies ${ }^{1 \dagger}$, Leonard N Chen ${ }^{1 \dagger}$, Aditi Bhagat ${ }^{1}$, Henry C Wright ${ }^{2}$, Sunghae Uhm, Joy S Kim', Thomas M Yung ${ }^{1}$, Siyuan Lei ${ }^{1}$, Gerald P Batipps², John Pahira², Kevin G McGeagh², Brian T Collins', Keith Kowalczyk², Gaurav Bandi², Deepak Kumar ${ }^{3}$, Simeng Suy ${ }^{1}$, Anatoly Dritschilo ${ }^{1}$, John H Lynch ${ }^{2}$ and Sean P Collins ${ }^{1 *}$

\begin{abstract}
Background: Erectile dysfunction after prostate radiation therapy remains an ongoing challenge and critical quality of life issue. Given the higher dose of radiation per fraction using stereotactic body radiation therapy (SBRT) there is concern that post-SBRT impotency would be higher than conventional radiation therapy approaches. This study sought to evaluate potency preservation and sexual function following SBRT for prostate cancer.

Methods: Between February 2008 and March 2011, 216 men with clinically localized prostate cancer were treated definitively with SBRT monotherapy at Georgetown University Hospital. Potency was defined as the ability to have an erection firm enough for intercourse with or without sexual aids while sexual activity was defined as the ability to have an erection firm enough for masturbation and foreplay. Patients who received androgen deprivation therapy (ADT) were excluded from this study. Ninety-seven hormone-naïve men were identified as being potent at the initiation of therapy and were included in this review. All patients were treated to 35-36.25 Gy in 5 fractions delivered with the CyberKnife Radiosurgical System (Accuray). Prostate specific antigen (PSA) and total testosterone levels were obtained pre-treatment, every 3 months for the first year and every 6 months for the subsequent year. Sexual function was assessed with the Sexual Health Inventory for Men (SHIM), the Expanded Prostate Index Composite (EPIC)-26 and Utilization of Sexual Medication/Device questionnaires at baseline and all follow-up visits.
\end{abstract}

Results: Ninety-seven men (43 low-, 50 intermediate- and 4 high-risk) at a median age of 68 years (range, 48-82 years) received SBRT. The median pre-treatment PSA was $5.9 \mathrm{ng} / \mathrm{ml}$ and the minimum follow-up was 24 months. The median pre-treatment total serum testosterone level was $11.4 \mathrm{nmol} / \mathrm{L}$ (range, $4.4-27.9 \mathrm{nmol} / \mathrm{L}$ ). The median baseline SHIM was 22 and $36 \%$ of patients utilized sexual aids prior to treatment. Although potency rates declined following treatment: 100\% (baseline); 68\% (6 months); 62\% (12 months); 57\% (18 months) and 54.4\% (24 months), 78\% of previously potent patients had erections sufficient for sexual activity at 24 months post-treatment. Overall sexual aid utilization increased from 36\% at baseline to $49 \%$ at 24 months. Average EPIC sexual scores showed a slow decline over the first two years following treatment: 77.6 (baseline); 68.7 (6 months); 63.2 (12 months); 61.9 (18 months); 59.3 (24 months). All sexual functions including orgasm declined with time. Prior to treatment, $13.4 \%$ of men felt their sexual function was a moderate to big problem which increased to $26.7 \%$ two years post treatment. Post-treatment testosterone levels gradually decreased with a median value at two year follow-up of $10.7 \mathrm{nmol} / \mathrm{L}$. However, the average EPIC hormonal scores did not illustrate a statistically significant difference two years post-treatment. Review of the radiation doses to the penile bulb in this study, a potential marker of post-treatment sexual function, revealed that the dose was relatively low and at these low doses the percentage of the penile bulb receiving 29.5 Gy did not correlate with the development of ED.

(Continued on next page)

\footnotetext{
* Correspondence: SPC9@georgetown.edu

${ }^{+}$Equal contributors

'Department of Radiation Medicine, Georgetown University Hospital, 3800

Reservoir Road, NW, Washington, DC 20007, USA

Full list of author information is available at the end of the article
}

\section{Biomed Central}

(c) 2013 Obayomi-Davies et al.; licensee BioMed Central Ltd. This is an open access article distributed under the terms of the Creative Commons Attribution License (http://creativecommons.org/licenses/by/2.0), which permits unrestricted use, distribution, and reproduction in any medium, provided the original work is properly cited. 
(Continued from previous page)

Conclusions: Men undergoing SBRT monotherapy for prostate cancer report sexual outcomes comparable to those reported for conventional radiation modalities within the first 24 months after treatment. Longer follow-up is required to confirm the durability of these findings.

Keywords: Prostate cancer, SBRT, CyberKnife, EPIC, Bother, Potency, Penile bulb

\section{Background}

Post-treatment sexual function is a primary determinant of satisfaction following prostate radiotherapy $[1,2]$. Erectile dysfunction (ED) occurs commonly following external beam radiation therapy (EBRT) and/or brachytherapy [3]. The incidence of ED is dependent on the potency definition $[4,5]$ and on the manner of data collection (i.e. patient or physician reported) [6]. Patients with radiation-induced impotence report a decrease in the quality and reliability of erection, ability to reach orgasm and overall ability to function sexually [7]. ED develops months to years after radiation therapy without recovery [2]. Patient characteristics such as a history of baseline ED [8], older age [9], obesity, comorbidities and pretreatment sexual aid usage may increase an individual's risk of radiation induced ED [10]. In addition, utilization of androgen deprivation therapy (ADT) [2] and radiationinduced hypogonadism [11] can adversely affect sexual outcomes while post-treatment utilization of sexual aids may enhance them [12-14].

The etiology of radiation induced ED is currently unclear but may involve damage to the neurovascular bundles (NVBs) [15], crura [16,17] and/or penile bulb (PB) $[16,18]$. Thus treatment-related factors such as the radiation dose to these adjacent sexual structures may contribute to the incidence and severity of ED. The risk of ED may be related to the volume of the crura/penile bulb in the high dose area [16]. To minimize ED, it is currently recommended that the median dose to the penile bulb be limited to $<50 \mathrm{~Gy}$ with conventionally fractionated EBRT [19]. Studies have shown that advanced radiation technologies such as intensity modulated radiation therapy (IMRT) may decrease the dose to the crura/penile bulb and potentially decrease ED [20,21].

The optimal radiation schedule for the treatment of prostate cancer is under active clinical investigation. Recent data suggest that large radiation fraction sizes are radiobiologically favorable over lower fraction sizes in prostate cancer $[22,23]$. The $\alpha / \beta$ for prostate cancer may be as low as $1.5 \mathrm{~Gy}$ [22]. If the $\alpha / \beta$ for prostate cancer is less than $3 \mathrm{~Gy}$, which is generally the value accepted for late sexual complications, the linear-quadratic model predicts that delivering large radiation fraction sizes will result in improved local control with a similar rate of sexual function complications.
SBRT is a safe and effective treatment for clinically localized prostate cancer [24-26]. The use of large fraction sizes in SBRT offers the potential radiobiological benefits of hypofractionation and potentially may minimize radiation-associated ED by reducing the volume of critical structures receiving high radiation doses. The CyberKnife robotic radiosurgical system uses image guidance to track implanted fiducials to account for intrafraction prostatic motion $[27,28]$. This reduces the uncertainty of the location of the prostate and allows treatment to be delivered with a smaller treatment volume expansion, which may reduce the doses delivered to the adjacent sexual structures. Initial reports suggest that the incidence of ED following SBRT is comparable to other radiotherapy modalities [29]. The goal of this study is to report the sexual outcomes following SBRT for clinically localized prostate cancer in previously potent men.

\section{Methods}

\section{Patient selection}

Patients eligible for this study were those who had histologically-confirmed prostate cancer and were potent (patient-reported response of "firm enough for intercourse" on Question 9 of the baseline EPIC-26) with or without sexual aids prior to treatment. Patients who received ADT were excluded from this study. Institutional IRB approval was obtained for retrospective review of data that was prospectively collected in our institutional database.

\section{SBRT treatment planning and delivery}

SBRT treatment planning and delivery were conducted as previously described [30]. Briefly, gold fiducials were placed into the prostate. Fused CT and MR images were used for treatment planning. The clinical target volume (CTV) included the prostate and the proximal seminal vesicles. The planning target volume (PTV) equaled the CTV expanded $3 \mathrm{~mm}$ posteriorly and $5 \mathrm{~mm}$ in all other dimensions. The prescription dose was 35-36.25 Gy to the PTV delivered in five fractions of 7-7.25 Gy over one to two weeks. The PB (proximal portion of the corpus spongiosum) was contoured [31] and evaluated with dose-volume histogram analysis during treatment planning using Multiplan (Accuray Inc., Sunnyvale, CA) inverse treatment planning. The dose-volume histogram (DVH) goal was for $<50 \%$ PB volume receiving 29.5 Gy. Assuming an $\alpha / \beta$ of 3 Gy for late sexual complications, this is 
Table 1 Baseline patient characteristics and treatment

\begin{tabular}{rrr}
\hline & Patients $(\mathbf{n}=\mathbf{9 7})$ \\
\hline Age (years) & Age $\leq 60$ & $14(14.4 \%)$ \\
$60<$ Age $\leq 70$ & $55(56.7 \%)$ \\
Age $>70$ & $28(28.9 \%)$
\end{tabular}

Race

Median Pre-Tx PSA

(ng/mL) Median

Median Pre-Tx

testosterone $(\mathrm{nmol} / \mathrm{L})$

Partner status

$$
\begin{array}{r}
\text { Partnered } \\
\text { Not partnered }
\end{array}
$$

Charlson comorbidity index (CCl)

0

Body mass index (BMI)

Risk group (D' Amico's)

Work status

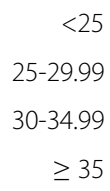

Low risk
Intermediate risk
High risk

Working

Non-working

Pre-treatment SHIM score

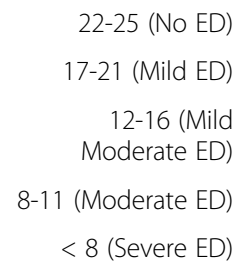

Sexual aid

$$
\begin{array}{r}
78(80.4 \%) \\
13(13.4 \%) \\
5(5.2 \%) \\
1(1 \%)
\end{array}
$$

$24(24.7 \%)$

$46(47.4 \%)$

$23(23.7 \%)$

$4(4.1 \%)$

$43(44.3 \%)$

$50(51.5 \%)$

4 (4.1\%)

$57(58.8 \%)$

$40((41.2 \%)$

$49(50.5 \%)$

$32(33.0 \%)$

$10(10.3 \%)$

$2(2.1 \%)$

*(No Sexual Activity) 4

$(4.1 \%)$

$62(63.9 \%)$

$35(36.1 \%)$

$1(1.0 \%)$

$0(0 \%)$
Table 1 Baseline patient characteristics and treatment (Continued)

\begin{tabular}{lrr}
\hline Dose (Gy) & \\
& 36.25 & $87(89.7 \%)$ \\
35 & $9(9.3 \%)$ \\
& Other & $1(1.0 \%)$ \\
\hline
\end{tabular}

biologically equivalent to approximately 50 Gy administered in 2 Gy fractions [32]. To minimize the risk of local recurrence, the dose to the NVBs was not restrained. Target position was verified during treatment using paired, orthogonal $x$-ray images.

\section{Follow-up and statistical analysis}

Prostate-specific antigen (PSA) and total testosterone levels were obtained before treatment and during routine follow-up visits every 3 months for the first year and every six months for the second year. Sexual function was assessed with the Expanded Prostate Index Composite (EPIC)-26 [7] and Utilization of Sexual Medication/Device questionnaires at baseline and at all follow-ups. The EPIC26 sexual function domain includes five questions related to sexual function and one question related to sexual bother (degree of interference or annoyance caused by limitations in sexual function) [33]. The sexual functions assessed included quality of erection (potency), ability to have erection, reliability of erections, ability to reach orgasm and overall ability to function sexually. The definition of potency and sexual activity were based on the patient-reported response to Question 9 on the EPIC-26. Potency was defined as the ability to have an erection firm enough for intercourse [34] while sexual activity was defined as the ability to have an erection firm enough for masturbation and foreplay [4].

Student's t-test and chi-square analysis were used to assess differences in ongoing PSA, testosterone and quality of life scores in comparison to baseline. Sample medians and ranges were used to describe continuous variables including PSA and testosterone. For each EPIC question, the responses were grouped into two to three clinically relevant categories. To statistically compare changes between time points, the levels of responses were assigned a score and the significance of the mean changes in the scores was assessed by paired $t$ test. EPIC scores for the sexual domain and its individual questions range from 0-100 with lower values representing worsening sexual symptoms. The minimally important difference (MID) in EPIC score was defined as a change of one-half standard deviation (SD) from the baseline [35]. To limit the effect of attrition bias, statistical analysis was limited to time points in which $\geq 80 \%$ of the patient data were available.

The impact of baseline patient characteristics on potency rates two years post-SBRT were evaluated by univariate 
Table 2 Quality of erection following SBRT for prostate cancer (patient-reported responses to question 9 of the EPIC-26)

\begin{tabular}{|c|c|c|c|c|c|c|c|}
\hline Follow up (months) & Start & 3 & 6 & 9 & 12 & 18 & 24 \\
\hline Firm enough for intercourse & $100.0 \%$ & $73.7 \%$ & $67.8 \%$ & $66.7 \%$ & $62.2 \%$ & $57.0 \%$ & $54.4 \%$ \\
\hline Firm enough for masturbation and foreplay only & $0.0 \%$ & $9.5 \%$ & $18.4 \%$ & $17.2 \%$ & $17.8 \%$ & $26.6 \%$ & $23.3 \%$ \\
\hline Not firm for any sexual activity & $0.0 \%$ & $16.8 \%$ & $13.8 \%$ & $16.1 \%$ & $20.0 \%$ & $16.5 \%$ & $22.2 \%$ \\
\hline$p$ & & $<0.0001$ & $<0.0001$ & $<0.0001$ & $<0.0001$ & $<0.0001$ & $<0.0001$ \\
\hline Total patient & 97 & 95 & 87 & 87 & 90 & 79 & 90 \\
\hline
\end{tabular}

and multivariate analyses. Univariate analysis of variance (ANOVA) was used to detect significant relationship between patient characteristics and potency at 2 years post treatment. In multivariate analysis, stepwise ordinal logistic regression modeling was used to determine independent factors predicting sexual function outcome. The baseline patient characteristics that were included as variables in the univariate and multivariant analyses included age, race, partner status, comorbidity, body mass index (BMI), risk group, work status, Sexual Health Inventory for Men (SHIM) score, erectile function and sexual aid usage. All tests were two-tailed, and a $p$ value $<0.05$ was considered significant. IBM ${ }^{\circledR}$ SPSS version 21 and MedCalc ${ }^{\circledR}$ version 12.6.1.0 were used to perform the statistical analyses.

\section{Results}

From February 2008 to March 2011, 216 prostate cancer patients were treated per our institutional SBRT monotherapy protocol. Ninety-seven men who were identified as being potent at the initiation of therapy and had a minimal follow up of two years post-treatment were included in this review (Table 1). They were ethnically diverse with a median age of 66.8 years (range, 4882 years). The median pre-treatment total serum testosterone level was $11.4 \mathrm{nmol} / \mathrm{L}$ (range, $4.4-27.9 \mathrm{nmol} / \mathrm{L}$ ). 43 patients were low-, 50 intermediate-, and 4 high-risk. 49.5\% patients had ED prior to treatment (baseline SHIM $\leq 21$ ) with a median baseline SHIM of 22 (range, $3-25) .36 .1 \%$ of patients were utilizing sexual aids prior to SBRT. Phosphodiesterase 5 (PDE5) inhibitors were utilized most commonly $(36 \%)$ followed by vacuum devices (1\%). No patients utilized transurethral suppositories or penile injections prior to treatment.

Ninety percent of patients were treated with 36.25 Gy in five 7.25 Gy fractions (Table 1). The median follow-up was 2.7 years. The median pre-treatment PSA of $5.9 \mathrm{ng} /$ $\mathrm{ml}$ declined to a median two years post-treatment of $0.5 \mathrm{ng} / \mathrm{ml}$. There was one biochemical failure, occurring in an intermediate-risk patient. The overall two-year actuarial biochemical relapse free survival was $99 \%$. No patient initiated ADT in the first two years following therapy.

Erections sufficient for intercourse declined following treatment: 100\% (baseline); $67.8 \%$ (6 months); $62.2 \%$
Table 3 Impact of baseline patient characteristics on potency rates two years post-SBRT

Characteristics

$\%$ Potent 2 years $p$ value

after SBRT

Age

$$
>65 \text { y/o pre-RT } \quad 47.4 \%
$$

Race

$\begin{array}{rrr}\text { White } & 51.2 \% & 0.574 \\ \text { Non-white } & 57.4 \% & \end{array}$

Partner status

$\begin{array}{rr}\text { Partnered } & 54.3 \% \\ \text { Non-partnered } & 55.0 \%\end{array}$

Charlson comorbidity index (CCI)

$\begin{array}{rrr}0 & 58.9 \% & 0.007^{*} \\ \geq 1 & 35.3 \% & \end{array}$

Body mass index (BMI)

$\begin{array}{lll}<30 & 60.9 \% & 0.943 \\ \geq 30 & 38.5 \%\end{array}$

Risk group (D' Amico's)

$\begin{array}{rrr}\text { Low risk } & 54.8 \% & 0.420 \\ \text { Intermediate-High Risk } & 54.2 \% & \end{array}$

Work status

$\begin{array}{rll}\text { Working } & 54.7 \% & 0.173 \\ \text { Non-working } & 54.1 \% & \end{array}$

Pre-treatment SHIM

$\begin{array}{lll}\geq 22 & 40.91 \% & 0.127 \\ <22 & 67.39 \% & \end{array}$

Sexual aid

$\begin{array}{rlr}\text { None } & 43.8 \% & 0.010^{* \#} \\ \text { Yes } & 60.3 \% & \end{array}$

SBRT Dose

$$
\begin{array}{rrr}
36.25 \text { Gy } & 50.0 \% & 0.933 \\
<36.25 \text { Gy } & 55.0 \% &
\end{array}
$$

*Significant in univariate analysis; \# Significant multivariate analysis. 
Table 4 Sexual aid utilization following SBRT for prostate cancer

\begin{tabular}{lcccc}
\hline $\begin{array}{l}\text { Sexual aid: } \\
\text { Follow-up }\end{array}$ & Oral & Vacuum & Suppository & Injection \\
\hline Start & $36.1 \%$ & $1.0 \%$ & $0.0 \%$ & $0.0 \%$ \\
$\mathbf{3}$ mon & $35.8 \%$ & $1.1 \%$ & $1.1 \%$ & $0.0 \%$ \\
$\mathbf{6}$ mon & $37.9 \%$ & $2.3 \%$ & $0.0 \%$ & $0.0 \%$ \\
$\mathbf{9}$ mon & $39.1 \%$ & $2.3 \%$ & $0.0 \%$ & $1.1 \%$ \\
$\mathbf{1 2}$ mon & $40.0 \%$ & $4.4 \%$ & $1.1 \%$ & $0.0 \%$ \\
$\mathbf{1 8}$ mon & $45.0 \%$ & 3.80025 & $1.3 \%$ & $1.3 \%$ \\
$\mathbf{2 4}$ mon & $48.9 \%$ & $3.8 \%$ & $2.2 \%$ & $2.2 \%$ \\
\hline
\end{tabular}

(12 months); 57\% (18 months) and 54.4\% (24 months) (Table 2$)$ which were statistically significant $(p<0.0001)$ at all time points. The decline in potency at two years was unlikely due solely to aging, as the average age of potent patients $(66.1 \mathrm{y} / \mathrm{o})$ was not statistically different from non-potent patients $(68.0 \mathrm{y} / \mathrm{o})(p=0.17)$. However, at two year post-SBRT, $77.8 \%$ of patients had erections that were satisfactory for sexual activity including masturbation and foreplay (Table 2). A Charlson Comorbidity Index of $\geq 1$ was significantly associated with a decreased probability of potency preservation at two years on univariate $(p=0.007)$, but was not significant on multivariate analysis $(p=0.246)$ (Table 3$)$. Sexual aid usage prior to SBRT was associated with an increased probability of potency preservation two years following treatment on both univariant $(p=0.010)$ and multivariant analysis $(p=0.037)$ (Table 3). No other baseline patient characteristics were significantly associated potency at two years following
SBRT. Two years post-treatment, overall sexual aid utilization increased to 48.9\%: oral medications (48.9\%), vacuum device (6.7\%), urethral suppository $(2.2 \%)$ and penile injection (2.2\%) (Table 4). No men utilized a penile prosthesis.

Adequate sexual function involves more than just possessing adequate erections [36]. The EPIC sexual domain allows for a more comprehensive and reliable assessment of a patients overall sexual function [37]. Average EPIC sexual scores showed a slow decline over the first two years following treatment: 77.6 (baseline); 68.7 (6 months); 63.2 (12 months); 61.9 (18 months); 59.3 (24 months) (Figure 1). At two year post-treatment, this change was statistically $(\mathrm{p}<0.001)$ and clinically significant (Figure 1$)$. All sexual functions including orgasm declined with time in a similar manner (Figure 2a, d, Table 5).

Sexual bother may be more important to an individual patient than sexual function. As for the overall EPIC sexual domain scores, post-SBRT sexual bother scores were significantly lower than pre-SBRT scores at all time points (Figure 3, Table 6). The proportion of men feeling that their sexual function was a moderate to big problem increased from $13.4 \%$ to $30 \%$ at 18 months post-SBRT (Figure 3, Table 6).

Pre-treatment total serum testosterone levels ranged from $4.4 \mathrm{nmol} / \mathrm{L}$ to $27.9 \mathrm{nmol} / \mathrm{L}$ with a median value of $11.4 \mathrm{nmol} / \mathrm{L} .24 .7 \%$ of patients were hypogonadal (total serum testosterone level below $8 \mathrm{nmol} / \mathrm{L}$ ) prior to SBRT. At two years the median serum testosterone value of $10.7 \mathrm{nmol} / \mathrm{L}$ (range, $2.5-26.4 \mathrm{nmol} / \mathrm{L}$ ) was not significantly lower than the pre-treatment value $(p=0.07)$ (Figure $4 \mathrm{a})$. The median absolute reduction was small $(0.7 \mathrm{nmol} / \mathrm{L})$

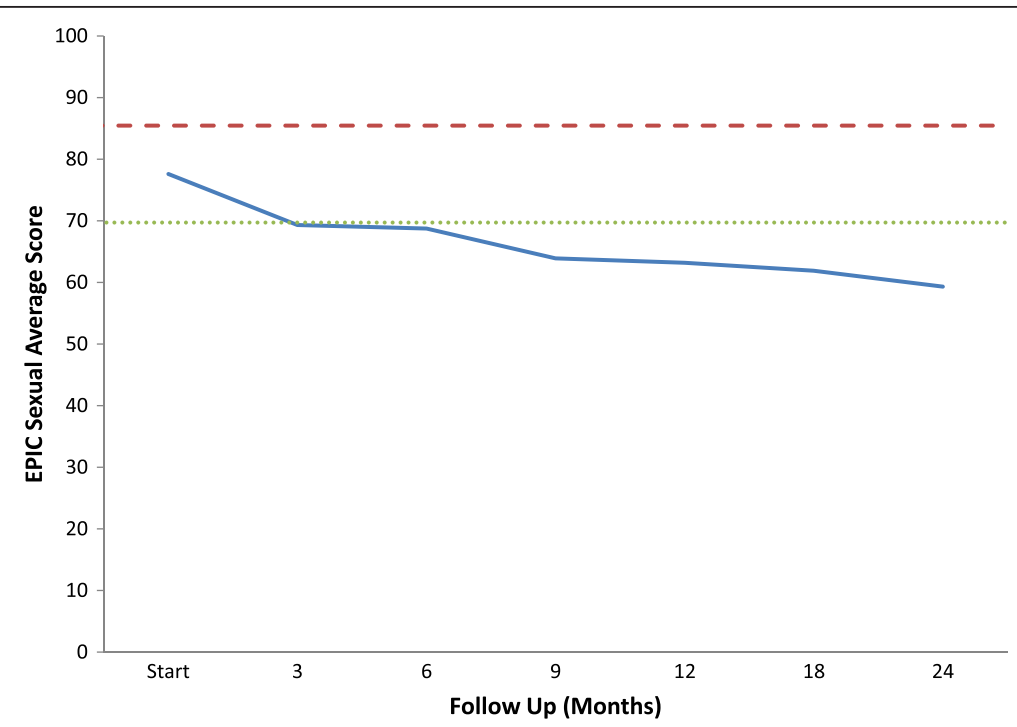

Figure 1 Average EPIC sexual domain scores at baseline and following SBRT for prostate cancer. Thresholds for clinically significant changes in scores ( $1 \frac{1}{2}$ standard deviation above and below the baseline) are marked with dashed lines. EPIC scores range from 0-100 with higher values representing a more favorable health-related QOL. 
and the median percent reduction was $5.8 \%$. The pretreatment and 2-year post SBRT biochemical hypogonadism rates were not significantly different (data not shown). At one month post-treatment, the mean EPIC hormone score declined to 93.5 from a baseline of $95.6(p=0.04)$; it returned to baseline by 6 months post-treatment (Figure $4 \mathrm{~b})$.

Similar to prior reports, the median PB volume in this series was $11 \mathrm{cc}$ [31]. The mean percentage of the $\mathrm{PB}$ receiving 29.5 Gy was $7.0 \%$ (range: $0-32.7 \%$ ), with the potent and impotent groups receiving 6.3\% (SD 6.5\%) and $7.2 \%$ (SD 8.6\%), respectively. The four patients who received 29.5 Gy to $>25 \%$ of the PB were impotent two years following treatment.

\section{Discussion}

Erectile dysfunction following prostate radiation therapy remains an ongoing challenge and critical quality of life issue [1]. Currently, there is limited data on sexual outcomes following SBRT for prostate cancer [29]. A better understanding of the sexual declines following SBRT would enable clinicians to provide more realistic expectations to patients as they weigh the complex treatment options [10]. Our prior report on sexual function following SBRT [30] utilized the SHIM questionnaire which primarily focuses on erectile function and is an inadequate measure of overall sexual function $[5,36]$. In this study, we utilized the EPIC-26 sexual function domain to evaluate erectile function but also assess overall sexual function including orgasmic function and sexual bother.

For this analysis, potency was rigorously defined as the ability to have an erection firm enough for intercourse [4]. Forty five percent of our patients were potent prior to SBRT and these patients were included in this analysis. Still, they were elderly with relatively low baseline SHIM scores and high pretreatment sexual aid utilization (Table 1). Even so, two years post-SBRT, $54 \%$ of these patients remained potent and $78 \%$ maintained an erection firm enough for masturbation and foreplay. As seen previously, the greatest decline in potency (a)

(c)
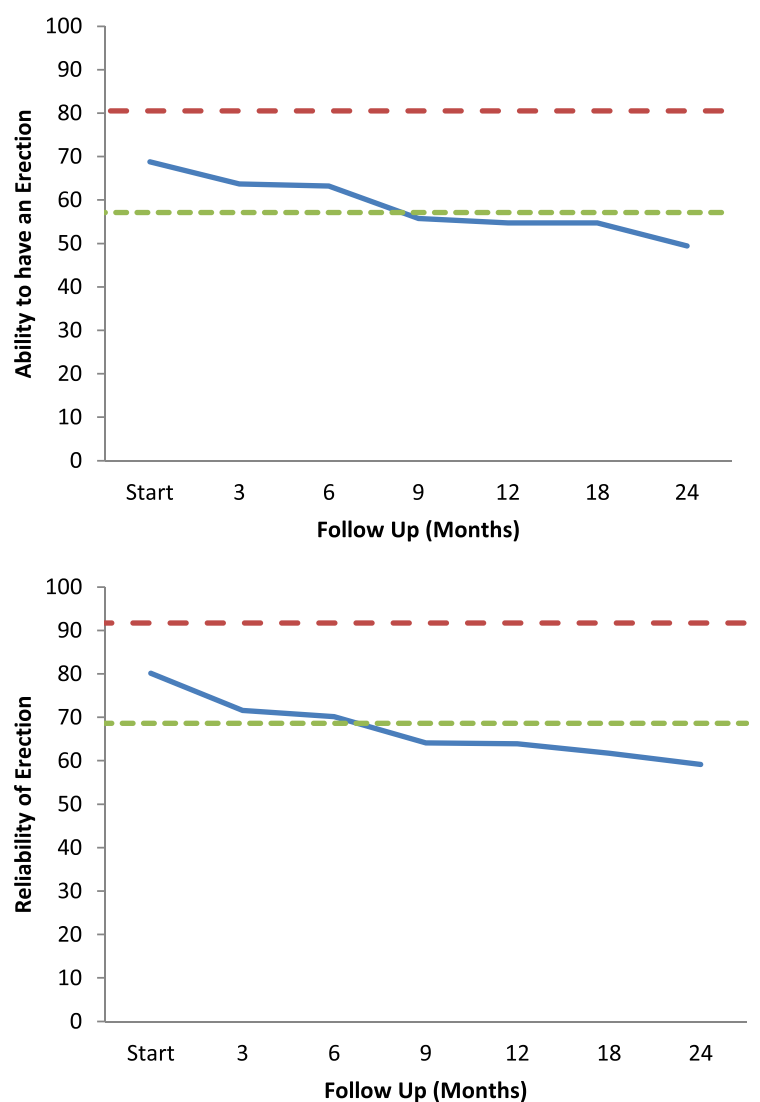

(b)

(d)
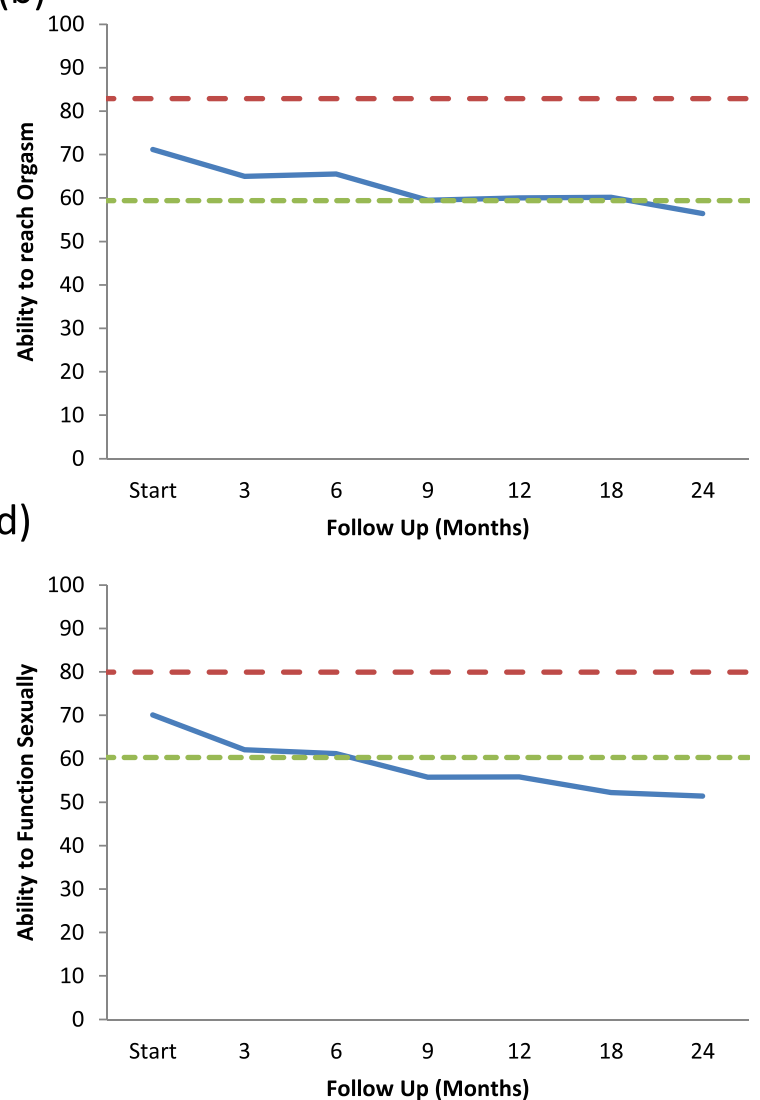

Figure 2 Average individual EPIC sexual function scores at baseline and following SBRT for prostate cancer. (a) ability to have an erection-Question 8A of the EPIC-26; (b) ability to reach orgasm- Question 8B of the EPIC-26; (c) reliability of erection- Question 10 of the EPIC-26; (d) ability to function sexually- Question 11 of the EPIC-26. Thresholds for clinically significant changes in scores (1/2 standard deviation above and below the baseline) are marked with dashed lines. EPIC scores range from 0-100 with higher values representing a more favorable health-related QOL. 
Table 5 Sexual functions following SBRT for prostate cancer: patient-reported responses to EPIC-26 questions 8A (ability to have an erection), 8B (ability to reach orgasm), 10 (reliability of erections) and 11 (ability to function sexually)

\begin{tabular}{|c|c|c|c|c|c|c|c|}
\hline & Start & 3 & 6 & 9 & 12 & 18 & 24 \\
\hline \multicolumn{8}{|l|}{ Ability to have an erection } \\
\hline Very good-good & $58.8 \%$ & $51.6 \%$ & $56.3 \%$ & $43.7 \%$ & $45.6 \%$ & $41.8 \%$ & $36.7 \%$ \\
\hline Fair & $34.0 \%$ & $33.7 \%$ & $26.4 \%$ & $26.4 \%$ & $25.6 \%$ & $29.1 \%$ & $31.1 \%$ \\
\hline Poor, very poor and none & $7.2 \%$ & $14.7 \%$ & $17.2 \%$ & $29.9 \%$ & $28.9 \%$ & $29.1 \%$ & $32.2 \%$ \\
\hline$p$ & & $<0.04$ & $<0.140$ & $<0.001$ & $<0.001$ & $<0.001$ & $<0.001$ \\
\hline \multicolumn{8}{|l|}{ Ability to reach orgasm } \\
\hline Very good-good & $74.2 \%$ & $56.8 \%$ & $63.2 \%$ & $51.7 \%$ & $53.3 \%$ & $53.2 \%$ & $46.7 \%$ \\
\hline Fair & $18.6 \%$ & $27.4 \%$ & $18.4 \%$ & $24.1 \%$ & $24.4 \%$ & $22.8 \%$ & $25.6 \%$ \\
\hline Poor, very poor and none & $7.2 \%$ & $15.8 \%$ & $18.4 \%$ & $24.1 \%$ & $22.2 \%$ & $24.1 \%$ & $27.8 \%$ \\
\hline$p$ & & $<0.05$ & 0.089 & $<0.003$ & $<0.001$ & $<0.001$ & $<0.001$ \\
\hline \multicolumn{8}{|l|}{ Reliability of erections } \\
\hline More than half-half the time & $78.4 \%$ & $66.3 \%$ & $64.4 \%$ & $55.2 \%$ & $57.8 \%$ & $54.4 \%$ & $52.2 \%$ \\
\hline Less than half the time & $21.6 \%$ & $33.7 \%$ & $35.6 \%$ & $44.8 \%$ & $42.2 \%$ & $45.6 \%$ & $47.8 \%$ \\
\hline$p$ & & $<0.004$ & $<0.003$ & $<0.001$ & $<0.001$ & $<0.001$ & $<0.001$ \\
\hline \multicolumn{8}{|l|}{ Function sexually (overall) } \\
\hline Very good-good & $63.9 \%$ & $53.7 \%$ & $52.9 \%$ & $47.1 \%$ & $47.8 \%$ & $43.0 \%$ & $40.0 \%$ \\
\hline Fair & $33.0 \%$ & $29.5 \%$ & $28.7 \%$ & $24.1 \%$ & $25.6 \%$ & $24.1 \%$ & $30.0 \%$ \\
\hline Poor-very poor & $3.1 \%$ & $14.7 \%$ & $16.1 \%$ & $28.7 \%$ & $26.7 \%$ & $32.9 \%$ & $30.0 \%$ \\
\hline$p$ & & $<0.003$ & $<0.005$ & $<0.001$ & $<0.001$ & $<0.001$ & $<0.001$ \\
\hline $\mathrm{N}=$ & 97 & 95 & 87 & 87 & 90 & 79 & 90 \\
\hline
\end{tabular}

following radiation therapy occurred in the first year with stabilization afterwards [38]. These results are comparable to results with conventionally fractionated EBRT or brachytherapy $[2,10,29]$.

The etiology of this early decline in potency is unclear but may involve exposure of the NVBs, crura or $\mathrm{PB}$ to excessive radiation. In this series, which included patients with unfavorable cancers, there was no attempt to spare the NVBs due to the concern that this may cause an unacceptably high rate of local failures [39]. Post-treatment sexual dysfunction may be exacerbated by high PB doses. With the aim of improving potency,

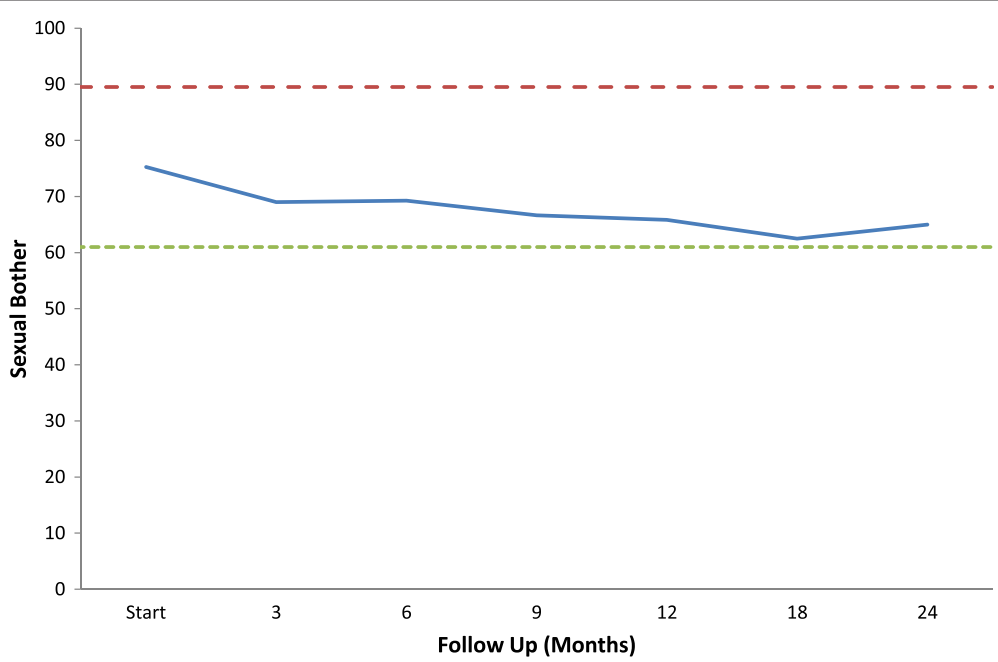

Figure 3 EPIC sexual bother at baseline and following SBRT for prostate cancer- Question 12 of the EPIC-26. Average sexual bother scores. Thresholds for clinically significant changes in scores ( $1 / 2$ standard deviation above and below the baseline) are marked with dashed lines. EPIC scores range from 0-100 with higher values representing a more favorable health-related QOL. 
Table 6 Sexual brother following SBRT for prostate cancer (patient-reported responses to question 12 of the EPIC-26)

\begin{tabular}{lccccccc}
\hline Sexual brother & Start & $\mathbf{3}$ mon & $\mathbf{6}$ mon & $\mathbf{9}$ mon & $\mathbf{1 2}$ mon & $\mathbf{1 8}$ mon & $\mathbf{2 4}$ mon \\
\hline No problem & $47.42 \%$ & $40.86 \%$ & $42.53 \%$ & $37.93 \%$ & $41.11 \%$ & $41.25 \%$ & $38.89 \%$ \\
Very small-small problem & $39.18 \%$ & $40.86 \%$ & $34.48 \%$ & $40.23 \%$ & $36.67 \%$ & $28.75 \%$ & $34.44 \%$ \\
Moderate-big problem & $13.40 \%$ & $18.28 \%$ & $22.99 \%$ & $21.84 \%$ & $22.22 \%$ & $30.00 \%$ & $26.67 \%$ \\
\hline
\end{tabular}

we have modified our institutional protocol. To reduce the PB dose, we have restricted the PB dose to $<25 \%$ of the volume receiving 29.5 Gy. Utilizing SBRT, this was easily achievable. PB doses below this did not correlate with impotence. We believe that such modifications have the potential to reduce the incidence and severity of impotence without increasing the risk of local failure.

Declining potency following SBRT suggests a need for approaches to improving long-term sexual outcomes. In this study, pre-treatment sexual aid usage was associated with increased potency two years following SBRT. Sexual aid utilization increased from a baseline of $37 \%$ to $49 \%$ at 24 months. Oral medication usage was common but vacuum device, urethral suppository, penile injection and/ or penile prosthesis were rarely utilized. It is not clear why post-treatment utilization of these potentially effective therapies [10] was not higher. Possible explanations include patient's perception of potential discomfort, the high cost of such treatments or partner availability. Others have shown that while sexual function may be improved by the use of sexual aids, sexual bother may be enhanced [33].

Like other treatment modalities, this study shows that SBRT impacts all areas of sexual function including erections, orgasm and overall satisfaction $[40,41]$. For example, only $46.7 \%$ of men two years following SBRT reported at least a good ability to reach orgasm compared to $74 \%$ prior to treatment (Table 5). The etiology of this decline in the ability to obtain orgasm is unclear but could be related to hemospermia, reduced ejaculate and/or painful ejaculation [42-45]. To better assess the etiology of orgasmic dysfunction, our future studies will employ an instrument to assess the effect of SBRT on ejaculation [46].

Bother may be a better indicator than function on how a treatment impacts an individual patient's quality of life [47]. Sexual bother is defined as the degree of interference or annoyance caused by limitations in sexual function and is dependent on an individual's pre-treatment erectile function, libido and partner status [33]. In general, previously potent men report a higher rate of bother following treatment than impotent men [48]. In this series of potent men, sexual bother slowly increased during the first two years following SBRT treatment (Figure 3, Table 6). Similar to other radiation modalities, this rate plateaued at 12-24 months following treatment with $30-40 \%$ of men reporting sexual bother as a moderate to big problem $[2,49]$. As in other series, sexual bother scores were better than sexual function scores.

Our study has several limitations. The penile bulb may not be the critical component of the erectile apparatus, but may be a surrogate for the crura in conventional radiation therapy treatment planning [16]. The utilization of non-coplanar beams in SBRT treatment could yield unexpectedly high doses to the crura [50]. Future studies should determine the crura dose during SBRT treatment and its impact on post-SBRT potency. In addition, the pretreatment utilization of sexual aids in our patients was high compared to previous reports [29]. The etiology is unclear but may limit the generalizability of this study.

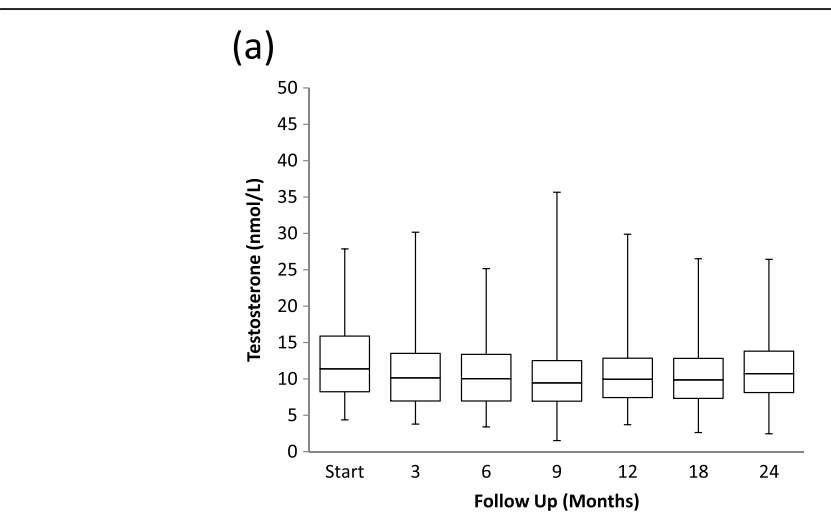

\section{(b)}

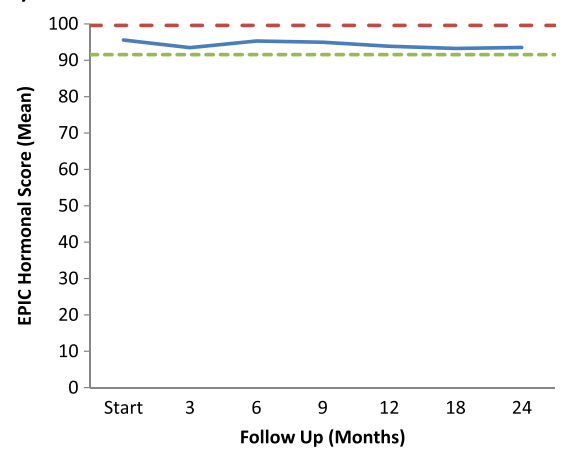

Figure 4 Pre- and post-treatment testosterone levels and EPIC hormonal scores. (a) Box-and-Whisker plot of total testosterone levels. The $p$ values were from $x^{2}$-analysis with baseline testosterone levels. (b) Average EPIC hormonal scores. Thresholds for clinically significant changes in scores ( $1 \frac{1}{2}$ standard deviation above and below the baseline) are marked with dashed lines. EPIC scores range from $0-100$ with higher values representing a more favorable health-related QOL. 


\section{Conclusions}

SBRT was well-tolerated for these patients with clinically localized prostate cancer. Men with preserved potency undergoing SBRT monotherapy for prostate cancer have sexual outcomes comparable to those reported for alternative radiation modalities within the first 24 months after treatment. Although most men retained sexual function, declining potency following treatment suggest a need for new approaches to improve long-term sexual outcomes.

\section{Abbreviations}

ADT: Androgen deprivation therapy; CTV: Clinical target volume; DVH: Dose-volume histogram; GTV: Gross target volume; NVBs: Neurovascular bundles; PB: Penile bulb; PTV: Planning target volume; QoL: Quality of life; SHIM: Sexual health inventory for men; EBRT: External beam radiation therapy; SBRT: Stereotactic body radiation therapy; EPIC: Expanded prostate index composite.

\section{Competing interests}

SP Collins and BT Collins serve as clinical consultants to Accuray Inc. The other authors declare that they have no competing interests.

\section{Authors' contributions}

$\mathrm{OO}$ and LC are lead authors, who participated in data collection, data analysis, manuscript drafting, table/figure creation and manuscript revision. $A B$ aided in the quality of life data collection and maintained the patient database. HW aided in the quality of life data collection and statistical analysis. SU aided in the quality of life data collection. JK aided in the quality of life data collection and maintained the patient database, aided in data collection, and participated in initial data interpretation. TY aided in the quality of life data collection and clinical data collection. SL is the dosimetrist who developed the majority of patients' treatment plans, and contributed to the dosimetric data analysis and interpretation. GB, JP, and KM aided in clinical data collection. BC, KK, GB, and DK participated in the design and coordination of the study. SS aided in statistical analysis, quality of life analysis and manuscript revision. AD is a senior author who aided in drafting the manuscript. $J \mathrm{~L}$ is a senior author who aided in drafting the manuscript. SC was the principal investigator who initially developed the concept of the study and the design, aided in data collection, drafted and revised the manuscript. All authors read and approved the final manuscript.

\section{Acknowledgements}

We acknowledge Daniel Hamstra, M.D., Ph.D. for helpful discussions,

\section{Author details}

'Department of Radiation Medicine, Georgetown University Hospital, 3800 Reservoir Road, NW, Washington, DC 20007, USA. 'Department of Urology, Georgetown University Hospital, Washington, DC, USA. ${ }^{3}$ Department of Biology, Cancer Research Laboratory, University of the District of Columbia, Columbia, Washington, DC, USA.

Received: 1 August 2013 Accepted: 24 October 2013

Published: 1 November 2013

\section{References}

1. Hoffman RM, Hunt WC, Gilliland FD, Stephenson RA, Potosky AL: Patient satisfaction with treatment decisions for clinically localized prostate carcinoma. Results from the Prostate Cancer Outcomes Study. Cancer 2003, 97:1653-1662.

2. Sanda MG, Dunn RL, Michalski J, et al: Quality of life and satisfaction with outcome among prostate-cancer survivors. N Engl J Med 2008, 358:1250-1261.

3. Robinson JW, Moritz S, Fung T: Meta-analysis of rates of erectile function after treatment of localized prostate carcinoma. Int J Radiat Oncol Biol Phys 2002, 54:1063-1068.

4. Krupski TL, Saigal CS, Litwin MS: Variation in continence and potency by definition. J Urol 2003, 170:1291-1294.

5. Schroeck FR, Donatucci CF, Smathers EC, et al: Defining potency: a comparison of the International Index of Erectile Function short version and the Expanded Prostate Cancer Index Composite. Cancer 2008, 113:2687-2694.
6. Sonn GA, Sadetsky N, Presti JC, Litwin MS: Differing perceptions of quality of life in patients with prostate cancer and their doctors. J Urol 2009, 182:2296-2302.

7. Szymanski KM, Wei JT, Dunn RL, Sanda MG: Development and validation of an abbreviated version of the expanded prostate cancer index composite instrument for measuring health-related quality of life among prostate cancer survivors. Urology 2010, 76:1245-1250.

8. Chen RC, Clark JA, Talcott JA: Individualizing quality-of-life outcomes reporting: how localized prostate cancer treatments affect patients with different levels of baseline urinary, bowel, and sexual function. J Clin Oncol 2009, 27:3916-3922.

9. Araujo AB, Mohr BA, McKinlay JB: Changes in sexual function in middleaged and older men: longitudinal data from the Massachusetts Male Aging Study. J Am Geriatr Soc 2004, 52:1502-1509.

10. Alemozaffar M, Regan MM, Cooperberg MR, et al: Prediction of erectile function following treatment for prostate cancer. Jama 2011, 306:1205-1214.

11. King CR, Maxim PG, Hsu A, Kapp DS: Incidental testicular irradiation from prostate IMRT: it all adds up. Int I Radiat Oncol Biol Phys 2010, 77:484-489.

12. Watkins Bruner D, James JL, Bryan CJ, et al: Randomized, double-blinded, placebo-controlled crossover trial of treating erectile dysfunction with sildenafil after radiotherapy and short-term androgen deprivation therapy: results of RTOG 0215. J Sex Med 2011, 8:1228-1238.

13. Raina R, Agarwal A, Goyal KK, et al: Long-term potency after iodine-125 radiotherapy for prostate cancer and role of sildenafil citrate. Urology 2003, 62:1103-1108

14. Pinkawa $M$, Gagel B, Piroth $M D$, et al: Erectile dysfunction after external beam radiotherapy for prostate cancer. Eur Urol 2009, 55:227-234.

15. DiBiase SJ, Wallner K, Tralins K, Sutlief S: Brachytherapy radiation doses to the neurovascular bundles. Int J Radiat Oncol Biol Phys 2000, 46:1301-1307.

16. van der Wielen GJ, Mulhall JP, Incrocci L: Erectile dysfunction after radiotherapy for prostate cancer and radiation dose to the penile structures: a critical review. Radiother Oncol 2007, 84:107-113.

17. van der Wielen GJ, Van Putten WL, Incrocci L: Sexual function after threedimensional conformal radiotherapy for prostate cancer: results from a dose-escalation trial. Int J Radiat Oncol Biol Phys 2007, 68:479-484.

18. Roach M 3rd, Nam J, Gagliardi G, El Naqa I, Deasy JO, Marks LB: Radiation dose-volume effects and the penile bulb. Int J Radiat Oncol Biol Phys 2010, 76:S130-S134

19. Mangar SA, Sydes MR, Tucker HL, et al: Evaluating the relationship between erectile dysfunction and dose received by the penile bulb: using data from a randomised controlled trial of conformal radiotherapy in prostate cancer (MRC RT01, ISRCTN47772397). Radiother Oncol 2006, 80:355-362.

20. Sethi A, Mohideen N, Leybovich L, Mulhall J: Role of IMRT in reducing penile doses in dose escalation for prostate cancer. Int J Radiat Oncol Biol Phys 2003, 55:970-978

21. Buyyounouski MK, Horwitz EM, Price RA, Hanlon AL, Uzzo RG, Pollack A: Intensity-modulated radiotherapy with MRI simulation to reduce doses received by erectile tissue during prostate cancer treatment. Int I Radiat Oncol Biol Phys 2004, 58:743-749.

22. Fowler JF: The radiobiology of prostate cancer including new aspects of fractionated radiotherapy. Acta Oncol 2005, 44:265-276.

23. Miles EF, Lee WR: Hypofractionation for prostate cancer: a critical review. Semin Radiat Oncol 2008, 18:41-47.

24. McBride SM, Wong DS, Dombrowski JJ, et al: Hypofractionated stereotactic body radiotherapy in low-risk prostate adenocarcinoma: preliminary results of a multi-institutional phase 1 feasibility trial. Cancer 2012, 118:3681-3690.

25. Freeman $D E$, King CR: Stereotactic body radiotherapy for low-risk prostate cancer: five-year outcomes. Radiat Oncol 2011, 6:3.

26. Katz AJ, Santoro M, Diblasio F, Ashley R: Stereotactic body radiotherapy for localized prostate cancer: disease control and quality of life at 6 years. Radiat Oncol 2013, 8:118

27. Hossain $\mathrm{S}$, Xia $\mathrm{P}$, Huang $\mathrm{K}$, et al: Dose gradient near target-normal structure interface for nonisocentric CyberKnife and isocentric intensity-modulated body radiotherapy for prostate cancer. Int J Radiat Oncol Biol Phys 2010, 78:58-63.

28. Xie Y, Djajaputra D, King CR, Hossain S, Ma L, Xing L: Intrafractional motion of the prostate during hypofractionated radiotherapy. Int J Radiat Oncol Biol Phys 2008, 72:236-246.

29. Wiegner EA, King CR: Sexual function after stereotactic body radiotherapy for prostate cancer: results of a prospective clinical trial. Int J Radiat Oncol Biol Phys 2010, 78:442-448. 
30. Chen LN, Suy S, Uhm S, et al: Stereotactic body radiation therapy (SBRT) for clinically localized prostate cancer: the Georgetown University experience. Radiat Oncol 2013, 8:58.

31. Wallner KE, Merrick GS, Benson ML, Butler WM, Maki J, Tollenaar BG: Penile bulb imaging. Int J Radiat Oncol Biol Phys 2002, 53:928-933.

32. Roach $M$, Winter K, Michalski JM, et al: Penile bulb dose and impotence after three-dimensional conformal radiotherapy for prostate cancer on RTOG 9406: findings from a prospective, multi-institutional, phase I/II dose-escalation study. Int J Radiat Oncol Biol Phys 2004, 60:1351-1356.

33. Litwin MS, Flanders SC, Pasta DJ, Stoddard ML, Lubeck DP, Henning JM: Sexual function and bother after radical prostatectomy or radiation for prostate cancer: multivariate quality-of-life analysis from CaPSURE. Cancer of the Prostate Strategic Urologic Research Endeavor. Urology 1999, 54:503-508.

34. NIH Consensus Conference. Impotence: NIH Consensus Development Panel on Impotence. Jama 1993, 270:83-90.

35. Norman GR, Sloan JA, Wyrwich KW: Interpretation of changes in healthrelated quality of life: the remarkable universality of half a standard deviation. Med Care 2003, 41:582-592.

36. Levinson AW, Ward NT, Sanda MG, et al: Comparison of validated instruments measuring sexual function in men. Urology 2010, 76:380-386.

37. Wei JT, Dunn RL, Litwin MS, Sandler HM, Sanda MG: Development and validation of the expanded prostate cancer index composite (EPIC) for comprehensive assessment of health-related quality of life in men with prostate cancer. Urology 2000, 56:899-905.

38. Siglin J, Kubicek GJ, Leiby B, Valicenti RK: Time of decline in sexual function after external beam radiotherapy for prostate cancer. Int J Radiat Oncol Biol Phys 2010, 76:31-35.

39. Ju AW, Wang H, Oermann EK: Hypofractionated stereotactic body radiation therapy as monotherapy for intermediate-risk prostate cancer. Radiat Oncol 2013, 8:30.

40. Hollenbeck BK, Wei JT, Sanda MG, Dunn RL, Sandler HM: Neoadjuvant hormonal therapy impairs sexual outcome among younger men who undergo external beam radiotherapy for localized prostate cancer. Urology 2004, 63:946-950.

41. Hollenbeck BK, Dunn RL, Wei JT, Montie JE, Sanda MG: Determinants of long-term sexual health outcome after radical prostatectomy measured by a validated instrument. J Urol 2003, 169:1453-1457.

42. Merrick GS, Wallner K, Butler WM, Lief JH, Sutlief S: Short-term sexual function after prostate brachytherapy. Int I Cancer 2001, 96:313-319.

43. Finney G, Haynes AM, Cross P, Brenner P, Boyn A, Stricker P: Cross-sectional analysis of sexual function after prostate brachytherapy. Urology 2005, 66:377-381.

44. Helgason AR, Fredrikson M, Adolfsson J, Steineck G: Decreased sexual capacity after external radiation therapy for prostate cancer impairs quality of life. Int J Radiat Oncol Biol Phys 1995, 32:33-39.

45. Huyghe $E$, Delannes $M$, Wagner $F$, et al: Ejaculatory function after permanent 125 I prostate brachytherapy for localized prostate cancer. Int J Radiat Oncol Biol Phys 2009, 74:126-132.

46. Rosen RC, Catania JA, Althof SE, et al: Development and validation of fouritem version of Male Sexual Health Questionnaire to assess ejaculatory dysfunction. Urology 2007, 69:805-809.

47. Gore JL, Gollapudi K, Bergman J, Kwan L, Krupski TL, Litwin MS: Correlates of bother following treatment for clinically localized prostate cancer. $J$ Urol 2010, 184:1309-1315.

48. Cooperberg MR, Koppie TM, Lubeck DP, et al: How potent is potent? Evaluation of sexual function and bother in men who report potency after treatment for prostate cancer: data from CaPSURE. Urology 2003, 61:190-196

49. Litwin MS, Gore JL, Kwan L, et al: Quality of life after surgery, external beam irradiation, or brachytherapy for early-stage prostate cancer. Cancer 2007, 109:2239-2247.

50. King CR, Lo A, Kapp DS: Testicular dose from prostate cyberknife: a cautionary note. Int J Radiat Oncol Biol Phys 2009, 73:636-637. author reply 637.

\section{doi:10.1186/1748-717X-8-256}

Cite this article as: Obayomi-Davies et al:: Potency preservation

following stereotactic body radiation therapy for prostate cancer.

Radiation Oncology 2013 8:256.

\section{Submit your next manuscript to BioMed Central and take full advantage of:}

- Convenient online submission

- Thorough peer review

- No space constraints or color figure charges

- Immediate publication on acceptance

- Inclusion in PubMed, CAS, Scopus and Google Scholar

- Research which is freely available for redistribution 